\title{
A Possible New Concept in the Mechanism of Action of Local Anesthesia
}

\author{
Maha T. Al-Saffar, Tahani A. AL-Sandook ${ }^{*}$, Mahmoud Y-Taha \\ Department of Dental Basic Science, College of Dentistry, Mosul University, Iraq \\ *Corresponding author: tahadent04@yahoo.com
}

Received October 20, 2013; Revised November 18, 2013; Accepted November 21, 2013

\begin{abstract}
Background: Local anesthetics act by different mechanisms to produce their effects in control acute and chronic pain. Methods: A total of 22 patients (14 female, 8 male) who need tooth apisectomy under infiltration local anesthesia were examined in two situations: Before and after administration of local anesthesia. For all patients salivary sample has been collected by salivette to estimate the level of opiorphin using ELISA Kits, the pain was assessed after administration of local anesthesia using Visual Analogue Scale (VAS). Results: the results showed a significant differences of salivary opiorphin before and after administration of local anesthesia $(5.96+5.38) \mathrm{ng} / \mathrm{ml}$ and $(14.49 \pm 3.66) \mathrm{ng} / \mathrm{ml}$ respectively with $\mathrm{p}<0.05$, the results also showed that the pain assessment by VAS was $(0.831 \pm 0.4587)$ with inverse correlation between the VAS and salivary opiorphin levels but with no significant difference ( $p>0.05$ ). Conclusions: local anesthetics (xylocaine $2 \%+$ adrenalin 1:80,000) used in apisectomy by infiltration technique produce a significant effects on salivary opiorphin $(\mathrm{p}<0.05)$ which may suggest a possible effects of local anesthetic on enkephalin pathway and the possibility of using this parameter for measurements of local anesthetic effects.
\end{abstract}

Keywords: salivary opiorphin, local anesthesia, dental pain

Cite This Article: Maha T. Al-Saffar, Tahani A. AL-Sandook, and Mahmoud Y-Taha, "A Possible New Concept in the Mechanism of Action of Local Anesthesia." American Journal of Medical and Biological Research 1, no. 4 (2013): 134-137. doi: 10.12691/ajmbr-1-4-5.

\section{Introduction}

Local anesthetics are believed to be the most frequently used drugs in clinical dentistry [1]. They are widely used to prevent or treat acute pain; to treat inflammatory, cancer related, and chronic pain [2], drugs classified as local anesthetics reversibly block action potential propagation in axons by preventing the sodium entry that produces the potentials [3]. Both nociceptive and neuropathic pain are targeted by this group of drug, and any part of the nervous system , from the periphery to the brain, may be where local anesthetic act to produce a desired anesthetic or analgesic effects: [4].

A

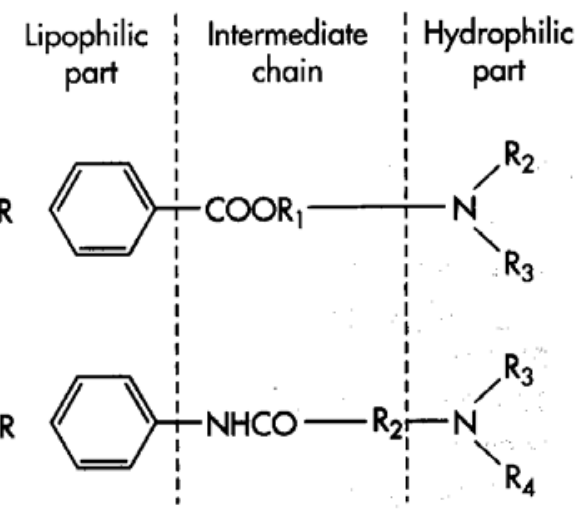

Figure 1. Typical local anesthetic. A: Ester type B: Amide type
All local anasthetics molecules in clinical use are classified chemically as amides and esters. These agents are weak bases, tertiary amines with three structures in common as shown in Figure 1:

1- Aromatic group- confers lipid solubility and allows nerve membrane penetration;

2- Intermediate chain - differentiates anesthetic as ester or amide.

3- Amino group- contributes water solubility which prevents precipitation of anesthetic [5].

opiorphin is an endogenous chemical compound first isolated from human saliva. It has pain killing effects greater than that of morphin and it works by stopping the normal break down of enkephalins, natural pain -killing opiod in spinal cord [6].

The visual analogue scale (VAS) measured on a scale of $0-10 \mathrm{~cm}$ anchored by two end - points indicating the two extremes: i.e "no ain" and "the worst possible pain" the VAS has been reported to be easily understood by patients, simple administer, reliable, and valid. The pain intensity measured with the single VAS is referred to as (recolled average) pain. The degree of intensity may be divided as mild $(0-3 \mathrm{~cm})$, moderate $(4-7 \mathrm{~cm})$, and sever (more than $7 \mathrm{~cm}$ ) $[7,8]$.

This study aims to compare the levels of opiorphin in saliva before and after the administration of infiltration local anesthesia for apisectomy and to estimate any possible correlation between the effects of dental local anesthesia (evaluated by VAS) and opiorphin level in saliva. 


\section{Patients, Materials and Methods}

This study was carried out at the specialized center for dentistry in Mosul city / Iraq, from February 2013 to July 2013. A total number of 22 patients were included in this study with an average age between (20-40) year old.

All patients require infiltration LA injection for dental treatment (Apisectomy) for maxillary teeth only, have no history of compromised medical status, no recent use of antibiotics or analgesic drugs (in the last one week), nonpregnant or lactating females, non- smoker, non alcoholic, and agreed to participate in this study and signed the consent form.

\subsection{Study Design and Sample}

1. Patient grouping: patients included in this study were examined in two situations:

a. Patients group required a dental treatment (Apisectomy) and were seated on a dental chair for dental and clinical examination but before taking LA injection, this group consisted of a total number of (22) patients (8 $\mathrm{M}, 14 \mathrm{~F}$ ), their mean of age (20-40) years.

b. patients group taking LA injection (infiltration) for dental treatments (Apisectomy), and consisted of a total number of (22) patients ( $8 \mathrm{M}, 14 \mathrm{~F}$ ), their mean of age (2040) year.

2. Drug used: Local anesthetic cartridge $1.8 \mathrm{ml}$ (lidocaine -hamein 2\%+1:80.000 adrenalin) MOH/IRAQ used to produce anesthesia by infiltration to maxillary teeth [3], the anesthesia were administrated by the researcher only for patients in a second situation only.

\subsection{Salivary Sample Collection and Storage}

Unstimulated saliva were collected for all patients before and after taking local anesthetic injection by 5-7 minute by asking the patients to rinse his or her mouth with $10 \mathrm{ml}$ of tap water to remove food debris. Then cotton-based techniques include using a simple cotton dental roll of specialized devices (Salivette), a cotton roll is sucked or chewed in patients mouth (cheek, floor of mouth, and over the tongue) for a 1 minute, this allows the saliva to be absorbed and collected in the swab in an easy and hygienic fashion, all sample should be collected between 9-11 am and should be clear of blood contamination:[9].

Salivette then centrifuged at $3000 \mathrm{rpm}$ for $10 \mathrm{~min}$, and the clear fluid sample at the bottom of tube is placed in sterile eppendroff tube and stored at $-20 \dot{\mathrm{C}}$ to be thawed for analysis [10].

\subsection{Subjective Measurement of Pain by VAS}

Patients after taking LA by 5-7 min rated their pain intensity using the horizontal visual analogue scale (VAS) an a $10-\mathrm{cm}$ line , labeled with anchor from" no pain" to "worst possible pain " as in Figure 2 [11].
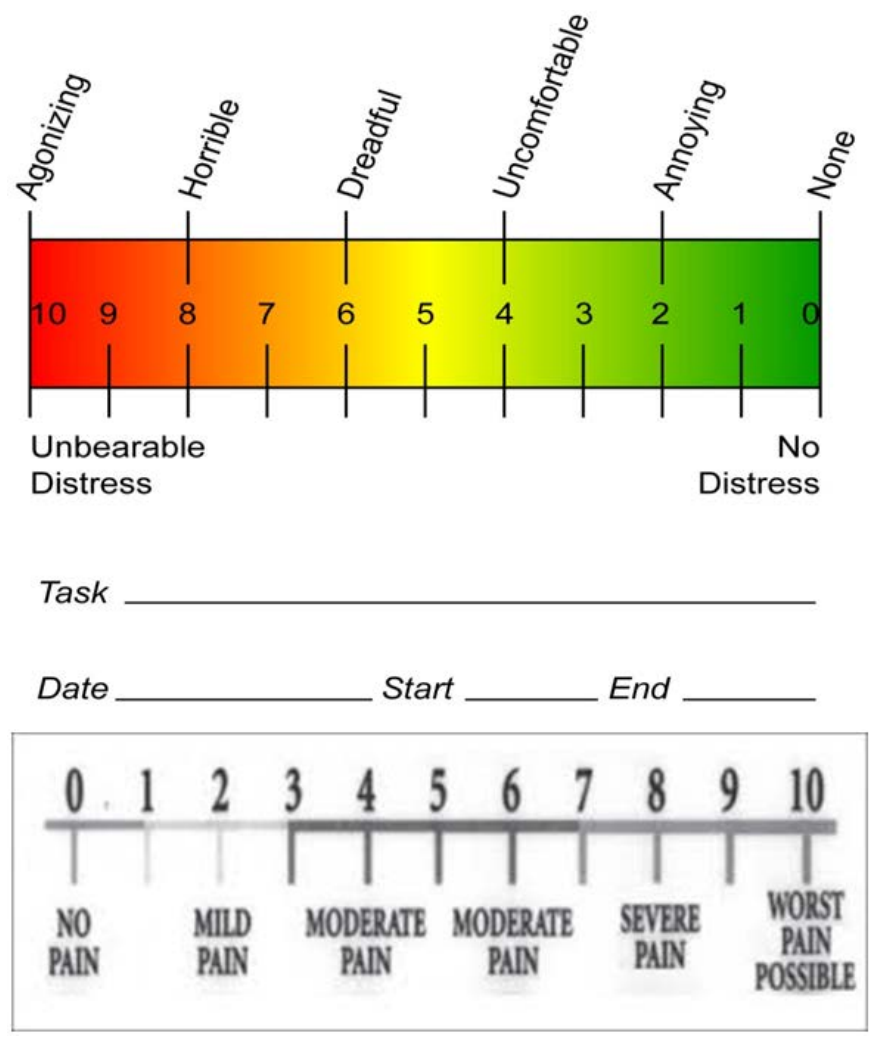

Figure 2. The visual analogue score (VAS)

\subsection{Human Opiorphin Measurement}

Measurement of human opiorphin was carried out using ELISA kit (MyBioSource, USA). The test is a quantitative sandwich enzyme immunoassay technique. Antibody specific for opiorphin has been pre-coated onto a microplate. The test was performed according to the manufacturer instructions.

\subsection{Data Analysis}

The data obtained from this study were subjected to the statistical analysis include descriptive and analytic 
methods. For descriptive way the mean of variance were used, while one way Analysis of variance and Duncan's Test for (intra - group comparison) and for (inter - group) matching with $5 \%$ level of significance, paired t-test had been used to compare between the studying group, and Kruskal Wallis test was used to determine the mean value of VAS while spearman test was used to estimate the possible correlation between the studying parameters.

\section{Results}

A total of 22 patients participated in this study. Most of them were females (63.6\% females, $36.4 \%$ males) as shown in Table1.

The mean age of samples was $25.5 \pm 6.68$ years for female and $29.5 \pm 3.5$ years for male with age range $20-40$ years (Table 2).
Table 1. Distribution of the study samples according to sex analyzed by ANOVA (one way analysis of variance)

\begin{tabular}{|c|c|c|}
\hline Sex & Number & Percentage \\
\hline Male & 8 & $(36.4)$ \\
\hline Female & 14 & $(63.6)$ \\
\hline Total & 22 & 100 \\
\hline
\end{tabular}

Table 2. The mean age and standard deviation of both sexes of the study group

\begin{tabular}{|c|c|c|c|}
\hline Sex & Mean of age \pm SD & Minim & Maximum \\
\hline Females & $25.5 \pm 6.68$ & 20 & 40 \\
\hline Males & $29.5 \pm 3.5$ & 20 & 40 \\
\hline
\end{tabular}

SD: standard deviation

The results of this study performed by paired t-test showed that the mean amount of opiorphin before taking local anesthesia was $(5.96+5.38) \mathrm{ng} / \mathrm{ml}$ while its amount was $(14.49 \pm 3.66) \mathrm{ng} / \mathrm{ml}$ in patients after taking local anesthesia with a significant differences between them (p $<.0 .05$ ) as shown in Table 3.

Table 3. Mean salivary opiorphin level pre and post - anesthetic administration analyzed by paired t-test

\begin{tabular}{|c|c|c|c|c|c|c|c|}
\hline Parameters & Before LA Mean \pm SD & After LA Mean+SD & Difference Mean \pm SD & $95 \%$ CI of difference & T-value & DF & P-value \\
\hline Opiorphin ng/ml & $5.96 \pm 5.38$ & $14.49 \pm 3.66$ & $-8.53 \pm 2.92$ & $-10.39-6.67$ & -10.105 & 25 & $<0.0001$ \\
\hline
\end{tabular}

The results of this study showed the measurement of VAS in patients after administration of local anesthesia performed by Kruskal Wallis test demonstrated that the mean value was $(0.831 \pm 0.4587) \mathrm{cm}$ in patients undergo Apisectomy of teeth (Table 4).

Table 4. The median and standard deviation of visual analogue scale (VAS) after administration of local anesthesia analyzed by Kruskal Wallis test

\begin{tabular}{|c|c|}
\hline Parameter & $\begin{array}{c}\text { Median } \pm \text { SD } \\
(\min -m a x)\end{array}$ \\
\hline VAS & $\begin{array}{c}0.831 \pm 0.4587 \\
\left(0 \_1.9\right)\end{array}$ \\
\hline
\end{tabular}

The spearman correlation test results showed that there are inverse correlation exist between VAS and salivary opiorphin level in patients who undergo apisectomy after administration of LA but with no significant difference between them ( $p>0.05)$ as shown in Table 5.

Table 5. Pearson correlation between visual analogue scale (VAS) and opiorphin level

\begin{tabular}{|c|c|}
\hline Parameter & VAS \\
\hline Opiorphin & $\mathbf{- 0 . 3 0}$ \\
& 0.4 \\
\hline
\end{tabular}

Note : upper bold fig (correlation coefficient r), lower figure (p-value), analysis were performed by spearman correlation test

\section{Discussion}

This study aims to compare the levels of opiorphin in saliva before and after the administration of infiltration local anesthesia for apisectomy and to estimate any possible correlation between the effects of dental local anesthesia(evaluated by VAS) and opiorphin level in saliva.

The results of this study showed that opiorphin level before and after administration of local anesthesia was ranged between (5.96-14.49) $\mathrm{ng} / \mathrm{ml}$ within the normal range of salivary opiorphin (2.8-25.9) $\mathrm{ng} / \mathrm{ml}$ [12].

The results of this study showed a significant effects of local anesthesia on opiorphin salivary levels which demonstrated by the different in the levels of salivary opiorphin between patients before and after administration of LA, Local anesthetics form the backbone of pain control techniques in dentistry. Local anesthetics are the safest and the most effective drugs in medicine for the prevention and management of pain [13]. There was no previous study on the possible relation between local anesthesia and salivary opiorphin levels. The possible explanation is that the temporary loss of sensation (produced by local anesthesia) including pain in one part of the body produced by a topically applied or injected agent without depressing the level of consciousness ${ }^{(14)}$. The nerve membrane is the site at which local anesthetics exert their pharmacologic actions. Many theories have been promulgated over the years to explain the mechanism of action of local anesthetics, including the acetylcholine, calcium displacement, and surface charge theories. Two other theories, membrane expansion and specific receptor, are given credence today [3]. Although pain is a subjective experience, there are two basic types of pain differing in their etiology and physiopathology: acute and chronic pain. Acute pain is self-limiting, usually concordant with the degree of on-going tissue damage, and remitting with resolution of the injury. It is nociceptive in nature. Chronic pain is not self-limiting, inadequately treated, and predominantly neuropathic in nature, leading to peripheral and/or central nociceptive sensitization:[15], Both types are affected by local anesthesia [2] and it was demonstrated in this study by subjective assessment of pain using VAS which showed mild pain score $(<2 \mathrm{~cm})$ for all patients.

The physiological opioid pathways are a predominant part of an endogenous nociceptive-modulating system that counterbalances the activity of pain transmission pathways. The most important endogenous opioid peptides, the enkephalins, play a major role in the dynamic control of pain perception [16]. Enkephalins interact with high affinity, with both the mu $(\mu)$ and delta (d)-opioid 
receptors, present on synaptic membranes of opioid and target neurons. Because of their high intrinsic efficacy, enkephalins need to occupy a smaller proportion of opioid receptors than morphine to elicit the same antinociceptive responses. Central administration of enkephalins appears to trigger a strong, but brief, analgesic responses due to their rapid inactivation by the concomitant action of two membrane-bound metallo-ectopeptidases which are colocated with opioid receptors, namely, neutral endopeptidase (NEP EC3.4.21.11) and aminopeptidase-N (AP-N EC3.4.11.2) [17]. Increasing the lifetime of circulating enkephalins, released in response to nociceptive stimuli, by inhibiting their degradation is, therefore, an effective method to increase their bioavailability and thus to enhance their physiological actions and particularly their analgesic potency [18].

One approach of new analgesic compounds relies on the potentiating of the enkephalin action by using small molecule inhibitors of their catabolism. The targets of this long pursued drug discovery concepts are the enkephalindegrading ectoenzyme aminopeptidaseN (APN) and neprilysin (NEP). Opiorphin ables to bind and inhibit both human aminopeptidase in vitro [19], Opiorphin inhibits three proteases: neutral ecto-endopeptidase (MME), ectoaminopeptidaseN (ANPEP) and perhaps also a dipeptidyl peptidase (DPP3), such action extends the duration of enkephalin effects where natural pain killers are released [20].

The increased levels of opiorphin during local anesthetic administration in this study may suggest possible relation between local anesthesia analgesic effects and enkephalin degredation pathway. A suggestion of future study is to evaluate the effects of different type of local anesthetics, and different methods of administration on opiorphin salivary level.

\section{Conclusion}

Local anesthetics (xylocaine 2\%+adrenalin 1:80,000) used in apisectomy by infiltration technique produce a significant effects on salivary opiorphin $(p<0.05)$ which may suggest a possible effects of local anesthetic on enkephalin pathway and possible new concept in its mechanism of action and the possibility of using this parameter for measurements of local anesthetic effects.

M.A. contributed to patient selection, data collection and writing up of the first draft of the paper T.A. contributed through preparing study design and data analysis and M.T. contributed to the immunological tests and analysis of results.

\section{References}

[1] Gaffen AS, Haas DA. Survey of local anesthetic use by Ontario dentists. JCDA 2009;75 (9):649.

[2] Heavner JE. Local anesthetics. Current Opinion in Anaesthesiology 2007; 20:336-342.

[3] Malamend ST. (2013). Technique of maxillary anesthesia In Malamed ST. Hand book of local anesthesia. $6^{\text {th }}$ edition .Mosby Elsevir Inc, New York, 2013.

[4] Shaban B, Moradi E, Nejat AH. Hemodynamic effect of $2 \%$ lidocaine with 1:80,000 epinephrin infiltration in maxillofacial surgeries under general anesthesia. JDMT 2013;2(1):17-20.

[5] Logothetis RM. (2011). Local anesthetic agent: A review of the current options for dental hygienistis. Journal of the California dental hygienists association 2011; 27(2):1-10.

[6] Dufour E, Villard-Saussine S, Mellon V. Opiorphin secretion pattern in healthy volunteers: gender difference and organ specificity. Biochem Anal Biochem 2013; 2(3):2-11.

[7] Monaco G, Dapril G, Tavern L. Mandibular third molar removal in young patients, an evaluation of 2 different flap designs. J Oral Maxillo Fac Surg, 2009; 67:15-21.

[8] Martin W, Heymans M, Skorpil N. Can a single pain rating replace a multiple pain rating in third molar surgery studies. Int $\mathrm{J}$ Oral Maxillo Fac Surg 2012; 41:1010-1013.

[9] Kanegane K, Penha S, Borsatti M. Dental anxiety in an emergency dental service.Rev Saude Publica 2003; 37:786-792.

[10] Nater YM, Rohleder N, Gaab J. Human salivary alpha - amylase reactivity in a psychosocial stress paradiagn. Int J Psychophysiol 2005; 55(3):333-341.

[11] Nayyar MS, Yates C. Bupivacaine as preemptive analgesia in third molar surgery, randomized control trail. Br J Oral Maxillo Fac Surg 2006; 44:501-503.

[12] Brkljacic LL, Sabalic MM, Salaric II. Development and validation of a liquid chromatography- tandem mass spectrometry method for the quantification of opiorphin in human saliva. J Chromatogr B Analyt Technol Biomed Life Sci 2011; 879 (32):3920-3926.

[13] Malamend SF. Buffering local anesthetics in dentistry. The pulse 2011; 44(1):8-9.

[14] American Academy of Pediatric Dentistry AADP. Clinical guide lines in guideline on use of local anesthesia for pediatric patient 2009; 34(6):182-186.

[15] Rougeot C, Robert F, Menz L. Systemically active human opiorphin is a potent yet non - addective analgesic without drug tolerance effects. Journal of Physiology and Pharmacology 2010; 61(4):483-490.

[16] Wisner A, Dufour E, Messaoudi M. Human opiorphin, a natural antinociceptive modulator of opiod- dependent pathways .PNAS 2006;103(47):17979-17984.

[17] Noble F, Banisadr G, Jardinand F. First discrete autoradiographic distribution of aminopeptidase $\mathrm{N}$ in various structures of rat brain and spinal cord using the selective iodinated inhibitor. Neuroscience 2001; 105:479-488.

[18] Noble F, Roques BP. Protection of endogenous enkephalin catabolism as natural approach to novel analgesic and antidepressant drug. Expert Opin Ther Trget 2007; 11:145-159.

[19] Pinto M, Rougeot C, Gracia L et. al. Proposed bioactive conformation of opiorphin, an endogenous dual APN/NEP inhibitor. Medicinal Chemistry 2012;3 (1):20-24.

[20] Thanawala V, Kadam VJ, Ghosh R. Enkephalin inhibitors: potential agents for the management of pain .Current Drug Targets 2008; 9(10):887-894. 